\title{
Colonic transendoscopic enteral tubing: A novel way of transplanting fecal microbiota
}

Authors

Institutions
Zhaoyuan Peng ${ }^{1,2}$, Jie Xiang ${ }^{1,2}$, Zhi He ${ }^{1,2}$, Ting Zhang ${ }^{1,2}$, Lijuan Xu ${ }^{1,2}$, Bota Cui ${ }^{1,2}$, Pan $\mathrm{Li}^{1,2}$, Guangming Huang ${ }^{1,2}$, Guozhong $\mathrm{ji}^{1,2}$, Yongzhan $\mathrm{Nie}^{3}$, Kaichun $\mathrm{Wu}^{3}$, Daiming Fan ${ }^{3}$, Faming Zhang ${ }^{1,2}$

Institutions are listed at the end of article. submitted

25. November 2015

accepted after revision

26. February 2016

\section{Bibliography}

DOI http://dx.doi.org/

10.1055/s-0042-105205

Published online: 28.4.2016

Endoscopy International Open

2016; 04: E610-E613

(c) Georg Thieme Verlag KG

Stuttgart · New York

E-ISSN 2196-9736

\section{Corresponding author}

\section{Faming Zhang, MD, PhD}

Medical Center for Digestive Diseases

The Second Affiliated Hospital of Nanjing Medical University 121 Jiang Jiayuan

Nanjing 210011

China

Fax: +86-25-58509883

fzhang@njmu.edu.cn
Background and study aims: Placement of a tube through the anus into the cecum has not yet been established as a method of administering whole-colonic treatment. The aim of this study was to evaluate the safety, feasibility, and value of transendoscopic enteral tubing (TET) for fecal microbiota transplantation (FMT) through the colon.

Patients and methods: A prospective observational study was performed of FMT using a new colonic TET technique. Under endoscopic guidance, a TET tube was affixed to the cecum with clips. The safety, value, and satisfaction with the FMT by TET were evaluated.

\section{Introduction}

$\nabla$

Fecal microbiota transplantation (FMT) has gained worldwide attention in recent years [1]. In 2013, it was recommended as the clinical choice in guidelines for treatment of recurrent Clostridium difficile infection (CDI) [2]. In addition, clinical studies have shown that FMT has a therapeutic role in inflammatory bowel diseases, refractory constipation, chronic diarrhea, and metabolic syndrome [3,4]. Since 2012, our group has been evaluating possible applications for and new methods of applying FMT [5-7].

Previous reports on FMT for delivery of treatment have involved the upper, middle, and lower gut [8-11]. FMT via colonoscopy is a classic approach, but patients have to endure bowel preparation and colonoscopy, especially when they need repeat treatment over a short period of time [12]. A traditional enema delivers solution only within the rectal and sigmoid colon which is way, in our previous studies of Crohn's disease and ulcerative colitis, why we chose to use FMT for delivery through the mid-gut $[6,7]$. However, patients may find it psychologically difficult to accept FMT through the upper and middle digestive
Results: A total of 54 patients underwent TET. The success rate of the TET procedure was 100\% (54/ 54). Duration of the TET procedures was $14.8 \pm$ $5.8 \mathrm{~min}$. During the TET tube retention period, $98.1 \%(53 / 54)$ of patients were satisfied with TET. The retention time for whole-colon delivery of the fecal microbiota suspension was $12.4 \pm 2.3$ days. In $88.4 \%$ (49/54) of cases, no discomfort was reported during injection through the TET tube of the microbiota suspension. No adverse events were see in patients who required tube extubation after FMT.

Conclusions: Colonic TET is a novel, safe, convenient, and reliable procedure for FMT that results in a high degree of patient satisfaction.

tract. Importantly, reflux and aspiration of bacterial liquid may occur and even cause asphyxia [13, 14]. There is currently no technique for placing a tube through the anus into cecum for whole-colon administration of treatment that could be maintained for repeat FMTs or while awaiting fresh fecal microbiota from the lab. Therefore, to solve these problems, we designed a new technique called transendoscopic enteral tubing (TET). As shown in Fig. 1, the TET tube is fixed to the cecum with clips under endoscopic guidance.

\section{Patients and methods}

$\nabla$

Subjects

A prospective observational study (NCT02560727) was conducted at the Second Affiliated Hospital of Nanjing Medical University from October 2014 to September 2015. All patients met the inclusion criteria, which were age 10 to 70 years, safety for the pilot study, suitability for endoscopy, and consent to undergo FMT and TET for their diseases and conditions. Patients were excluded it they had severe bowel lesions 


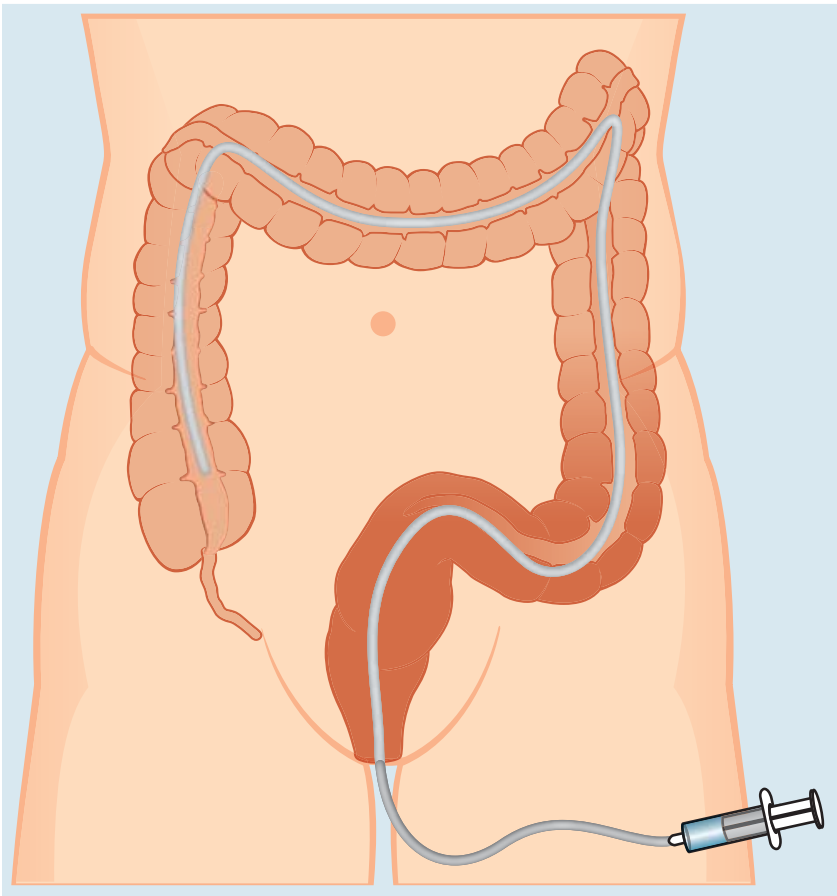

Fig. 1 Concept sketch of FMT treatment by TET.

with stenosis, fistula, or the risk of perforation; complex perianal lesions or serious lesions in the ileocecal junction or ascending colon; and no proper site for titanium clip fixation. Informed consent was obtained from all adult subjects or parents in pediatric cases. The study was approved by the Institutional Review Board of the Second Affiliated Hospital of Nanjing Medical University.

\section{Preparation, procedures and assessment of TET}

Regular colonoscopy was performed under intravenous anesthesia. After examination and evaluation of the whole colon, the TET tube (FMT medical, Nanjing, China) was inserted into the ileocecal junction through the endoscopy channel ( $\bullet$ Fig.2). The colonoscope was removed from the colon while the TET tube was maintained at the ileocecal junction. Then the colonoscope was inserted into the ileocecum again. The line circle on the TET tube was affixed to the intestinal wall using titanium clips under direct vision (generally two titanium clips at the first station and one to two clips at the second and/or third station as necessary) (- Fig. 3 and - Fig.4). Next the colonoscope was withdrawn carefully and slowly. The distal tube was affixed to the skin of the buttocks (preferably on the left side) with medical adhesive plaster. A valve was connected to the terminal TET tube. The procedure time and all related adverse events (AEs) were recorded. According to the concept shown in $\bullet$ Fig. 1, the patient was required to be in the right-lateral position and then $200 \mathrm{~mL}$ of suspension was injected through the TET tube. The 200-mL suspension was a mixture of $150 \mathrm{~mL}$ saline and $50 \mathrm{~cm}^{3}$ centrifuged microbiota that was purified following our lab protocol and using the automatic system GenFMTer (FMT medical, Nanjing) [6,7]. The duration of the injection was recorded and intended to be more than 1 minute so as to avoid the abdominal discomfort that would be associated with a quicker procedure. After FMT, patients were required to remain in the right-lateral position for 30 minutes. Retention of the microbiota suspension for over 1 hour indicated successful delivery of the microbiota through colonic TET. In some cases, FMT was repeated during subsequent

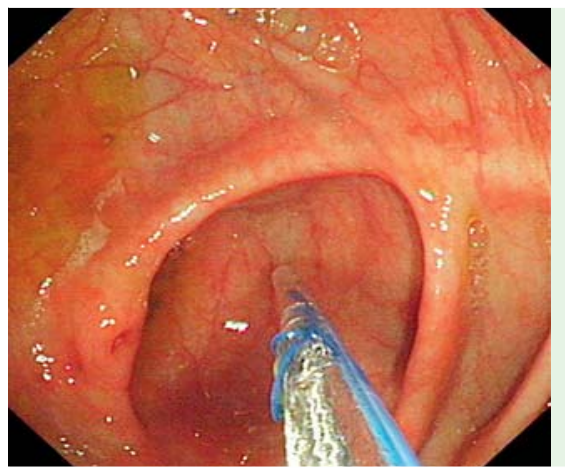

Fig. 2 TET tube inserted into cecum through endoscopic channel.

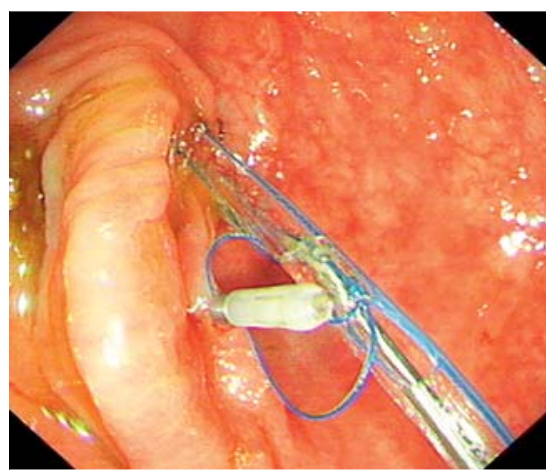

Fig.3 Attachment of titanium clips to the TET tube to the cecum mucosa at the first station under endoscopic guidance. The tip of the guidewire can be seen within the tube.

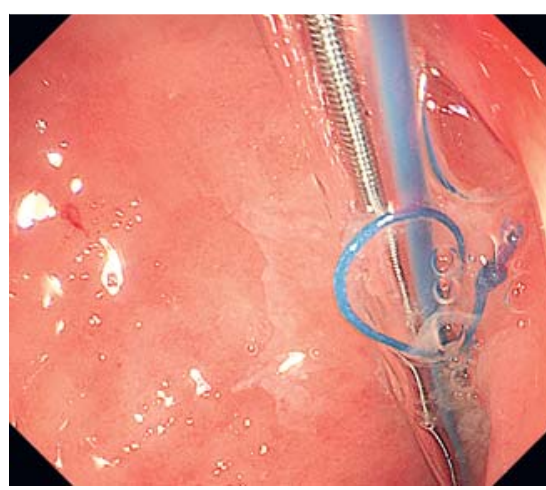

Fig. 4 The second station of TET tube attachment in the ascending colon mucosa with titanium clips under endoscopic guidance. The guidewire can be seen within the tube.

days to ensure infusion of the microbiota to the whole colon. Reports from patients of discomfort during FMT were recorded and all of them agreed to participate in the post-treatment survey on satisfaction with TET and FMT.

\section{Results}

$\nabla$

As shown in Table 1, 54 patients were included in this prospective study: 32 males and 22 females aged 10 to 70 years (mean \pm $\mathrm{SD}, 34.5 \pm 10.4$ ). Of the patients, 23 cases had ulcerative colitis, 16 had Crohn's disease, five had unexplained chronic diarrhea, five had constipation, three had small intestinal bacterial overgrowth, and 2 had irritable bowel syndrome. TET was successful in all 54 cases (100\%). The mean time from advancement of the TET tube through the channel to the end of the tubing was 14.8 minutes. Three clips were used on two fixation stations of the TET tube in 36 cases during our preliminary observational period or in patients who only needed short-term retention of TET tube retaining. In the remaining 15 cases, four clips were used on two fixation stations. No complications such as mild to serious abdominal pain and bleeding stool were observed. During the TET tube retention period, $98.1 \%$ of patients $(53 / 54)$ were satisfied with TET; one patient with ulcerative colitis complained of tolerable 
Table 1 Characteristics of patients undergoing FMT through colonic TET.

\begin{tabular}{|l|l|}
\hline Characteristics & N (\%) \\
\hline Total number & $54(100)$ \\
\hline Age, mean \pm SD, years & $34.5 \pm 10.4$ \\
\hline Male & $32(59)$ \\
\hline Disease category & $23(42.5)$ \\
\hline Ulcerative colitis & $16(29.6)$ \\
\hline Crohn's disease & $5(9.3)$ \\
\hline Unexplained chronic diarrhea & $5(9.3)$ \\
\hline Constipation & $3(5.6)$ \\
\hline Small intestinal bacterial overgrowth & $2(3.7)$ \\
\hline Irritable bowel syndrome & $54(100)$ \\
\hline TET success rate, $n$ (\%) & $14.8 \pm 5.8$ \\
\hline Tubing time, mean \pm SD, min & $12.4 \pm 2.3$ \\
\hline Retention time of tube, mean \pm SD, days & \\
\hline Removal of tube & $35(64.8)$ \\
\hline Tube falling out & $19(35.2)$ \\
\hline Tube extraction & $53(98.1)$ \\
\hline Satisfaction survey for FMT via TET
\end{tabular}

FMT, fecal microbiota transplantation; TET, transendoscopic enteral tubing; $\mathrm{SD}$, standard deviation.

mild anal discomfort; no other patients complained of anal pendant expansion.

A total of $200 \mathrm{~mL}$ of fecal microbiota suspension was given to each patient and the mean injection time was 3.2 minutes. In all cases (100\%), FMT through colonic TET was successful. In $88.4 \%$ of cases (49/54), patients reported no discomfort during FMT. One patient (1.9\%) with severe ulcerative colitis complained of abdominal pain after infusion through TET tube, and the symptoms were alleviated with rest. Four patients (7\%) reported tolerable mild abdominal distension. In one case, we encountered difficult with the infusion because of bending of the tube at an angle in the intestines, which was resolved with injection of normal saline at high pressure. The time from the end of the FMT to the first defecation was $6.3 \pm 0.7$ hours. In 35 patients with ulcerative colitis or Crohn's disease, the tubes fell off. Mesalazine was delivered daily to them through the TET tube until the tube fell off. The retention duration was $12.4 \pm 2.3$ days. In 19 patients, removal of the TET tube was required after FMT or colonic administration. No AEs were encountered when the tubes fell off or on removal of them. All clips were observed on the TET tubes after their removal from the colon.

\section{Discussion \\ $\nabla$}

Reports exist of use of FMT to deliver treatment to the upper gut, mid-gut and lower gut. Oral intake of fecal microbiota capsules is common through the upper gut [1]. The capsule dissolves in the small intestine and bacteria are distributed in the gastrointestinal tract. In mid-gut, the microbiota suspension is injected into the small intestine below the second duodenal segment under endoscopic direct vision or via a nasogastric tube, small intestine stoma or percutaneous endoscopic gastrostomy with jejunal extension (PEGJ)[15]. Fecal microbiota can be delivered to the lower gut through colonoscopy, enema, distal ileum stroma, colostomy, and colonic TET. The advantages and limitations of different methods of delivering FMT are shown in Table 2.

In this study, TET and FMT were successfully performed in all 54 cases (100\%), and FMT retention time was longer than 1 hour. This TET technique and the novel TET accessary devices used demonstrate the feasibility and significance of colonic administration. No serious AEs were observed during the TET procedure, infusion of FMT through the TET tube, the period during which the TET tube was left in place, or removal of the device. During the FMT procedure, $90.7 \%$ (49/54) of patients reported no discomfort. The mean retention time for the microbiota suspension was enough to meet the requirement for FMT according to our protocol. For patients who required repeat FMT and combined colonic administration of mesalazine, it was a convenient and economic way to use the colonic TET tube. Importantly, this method may be less psychologically challenging for patients than delivery of FMT via the upper and middle digestive tract.

Table 2 Current ways of delivering FMT

\begin{tabular}{|c|c|c|}
\hline Method of delivery & Advantages & Limitations \\
\hline \multicolumn{3}{|l|}{ Upper-gut } \\
\hline Oral capsules[9] & Convenience for patients; easy to deliver & $\begin{array}{l}\text { Efficacy affected by cryopreservation state [3]; bacteria possibly af- } \\
\text { fected by bile salts [5] and cryopreservation state; potential risk of SIBO }\end{array}$ \\
\hline \multicolumn{3}{|l|}{ Mid-gut } \\
\hline Endoscopic channel & Easy to deliver; easy to maintain & $\begin{array}{l}\text { Only used during endoscopy; reflux and aspiration [4]; bacteria possi- } \\
\text { bly affected by bile salts [5] and cryopreservation; potential risk of } \\
\text { SIBO; not convenient to repeat }\end{array}$ \\
\hline Nasojejunal tube [5] & Easy to deliver; easy to maintain & $\begin{array}{l}\text { Only used in patients with nasojejunal tube; bacteria possibly affected } \\
\text { by bile salts; potential risk of SIBO }\end{array}$ \\
\hline PEGJ tube & Easy to deliver; easy to maintain & $\begin{array}{l}\text { Only used in few patients with PEG] tube; bacteria possibly affected by } \\
\text { bile salts; potential risk of SIBO }\end{array}$ \\
\hline \multicolumn{3}{|l|}{ Lower-gut } \\
\hline Endoscopic channel & Easy to deliver & $\begin{array}{l}\text { Only used during colonoscopy; difficult to hold the infused suspension } \\
\text { in colon; not convenient to repeat }\end{array}$ \\
\hline Traditional enema & Easy to deliver; low cost & $\begin{array}{l}\text { Only cover rectosigmoid colon; limited infused volume; not suitable for } \\
\text { patients having difficulty to hold the infused suspension in rectum }\end{array}$ \\
\hline Stoma in ileocolon & $\begin{array}{l}\text { Convenient to repeat FMT; easy to deliver; avoiding } \\
\text { bacteria affected by bile salts; easy to maintain }\end{array}$ & $\begin{array}{l}\text { Only used in few patients with PEC [15] tube or surgical double cavity } \\
\text { stoma formation in ileocolon }\end{array}$ \\
\hline Colonic TET tube & $\begin{array}{l}\text { Convenient to repeat FMT; easy to deliver; avoiding } \\
\text { bacteria affected by bile salts; easy to maintain }\end{array}$ & TET tube must be placed under colonoscopy \\
\hline
\end{tabular}

FMT: fecal microbiota transplantation; PEG]: percutaneous endoscopic gastrostomy with jejunal extension; SIBO: small intestinal bacterial overgrowth; TET: transendoscopic enteral tubing. 
In 19 patients, the TET tubes were removed after a single FMT or colonic administration. Of them, 4 with IBD required removal of the TET tube after FMT and 1-week administration of mesalazine (7 enemas in a single package of Salofalk) because there was no need to leave the TET tube in place. According to our lab protocol and clinical flow [6,7], for consideration of improving possible clinical efficacy, the time from defecation of stool to infusion of microbiota was required to be no more than 1 hour. Therefore, 15 patients agreed to the use of TET while waiting for fresh microbiota from the lab and because they preferred psychologically delivery through the lower gut, and their TET tubes were removed after FMT.

It should be emphasized that the patients' daily lives were not affected by TET, and in $98.1 \%$ of cases (53/54), they were satisfied with FMT through TET. For patients with ulcerative colitis that involved the entire colon or Crohn's disease, TET should be a wonderful choice for frequently delivering medication into the whole colon. In the current study, all 23 patients with ulcerative colitis and 16 patients with Crohn's disease were administered a mesalazine enema through the TET tube. Of them, six patients were discharged with the tube and they were able to conveniently infuse medication by themselves at home.

The novel concept of TET may be promising for intestinal interventional therapy. Besides this pilot study on colonic study for whole-colon administration through a colonic TET tube, a study on the similar concept of TET used for interventional therapy by duodenal or jejunal TET under gastroscopy is ongoing in our center.

This study does, however, have some limitations. The sample size of this pilot study was small, but a larger prospective study based on these preliminary results is ongoing. This study did not evaluate clinical responses to whole-colon administration compared with other traditional treatments; that will be part of our future studies. A cost-efficacy analysis is necessary and the results may vary from country to country depending on policies for medical charges. Patients who require only a single FMT (e.g. CDI) may not need to generally may not need to undergo colonic TET if use of fresh fecal microbiota is not a consideration and that is why there were no CDI patients in this study.

In conclusion, this study is the first report of colonic TET as a convenient and safe way of delivering FMT. The results highlight the significance of colonic TET as a promising technique for single and repeat whole-colon administration of medication.

Competing interests: Dr. Zhang invented the concept of transendoscopic enteral tubing and devices related to it.

\section{Institutions}

${ }^{1}$ Medical Center for Digestive Diseases, the Second Affiliated Hospital of Nanjing Medical University, Nanjing 210011, Jiangsu Province, China.

${ }^{2}$ Key Lab of Holistic Integrative Enterology, Nanjing Medical University, 121 Jiangjiayuan, Nanjing 210011, Jiangsu Province, China.

${ }^{3}$ State Key Laboratory of Cancer Biology \& Xijing Hospital of Digestive

Diseases, the Fourth Military Medical University, Xi'an 710032, China.

\section{Acknowledgements}

$\nabla$

This research was supported by public donated Intestine Initiative Foundation, Clinical Science and Technology Foundation of Jiangsu Province (BL2014097), and National Gastroenterology Research Project (2015BAI13B07).

\section{References}

1 Kamada N, Seo SU, Chen GY et al. Role of the gut microbiota in immunity and inflammatory disease. Nat Rev Immunol 2013; 13: 321 - 335

2 Surawicz CM, Brandt LJ, Binion DG et al. Guidelines for diagnosis, treatment, and prevention of Clostridium difficile infections. Am J Gastroenterol 2013; 108: $478-499$

3 Damman CJ, Miller SI, Surawicz CM et al. The microbiome and inflammatory bowel disease: is there a therapeutic role for fecal microbiota transplantation. Am J Gastroenterol 2012; 107: 1452 - 1459

4 van Nood E, Vrieze A, Nieuwdorp $M$ et al. Duodenal infusion of donor feces for recurrent Clostridium difficile. N Engl J Med 2013; 368: $407-415$

5 Zhang FM, Wang HG, Wang $M$ et al. Fecal microbiota transplantation for severe enterocolonic fistulizing Crohn's disease. World J Gastroenterol 2013; 19: 7213-7216

6 Cui B, Li P, Xu L et al. Step-up fecal microbiota transplantation strategy: a pilot study for steroid-dependent ulcerative colitis. J Transl Med 2015; 13: 298

7 Cui B, Li P, Xu L et al. Fecal microbiota transplantation is an effective rescue therapy for refractory inflammatory bowel disease. Inflamm Cell Signal 2015; 2: e757

8 Cui B, Feng $Q$, Wang $H$ et al. Fecal microbiota transplantation through mid-gut for refractory Crohn's disease: safety, feasibility, and efficacy trial results. J Gastroenterol Hepatol 2015; 30: 51 - 58

9 Youngster I, Russell GH, Pindar C et al. Oral, capsulized, frozen fecal microbiota transplantation for relapsing Clostridium difficile infection. JAMA 2014; 312: $1772-1778$

10 Moayyedi P, Surette MG, Kim PT et al. Fecal microbiota transplantation induces remission in patients with active ulcerative colitis in a randomized controlled trial. Gastroenterology 2015; 149: 102-109

11 Rossen NG, Fuentes S, van der Spek MJ et al. Findings from a randomized controlled trial of fecal transplantation for patients with ulcerative colitis. Gastroenterology 2015; 149: 110-118

12 Angelberger S, Reinisch W, Makristathis A et al. Temporal bacterial community dynamics vary among ulcerative colitis patients after fecal microbiota transplantation. Am J Gastroenterol 2013; 108: 1620-1630

13 Anderson JL, Edney RJ, Whelan K. Systematic review: faecal microbiota transplantation in the management of inflammatory bowel disease. Aliment Pharmacol Ther 2012; 36: $503-516$

14 Colman RJ, Rubin DT. Fecal microbiota transplantation as therapy for inflammatory bowel disease: a systematic review and meta-analysis. J Crohns Colitis 2014; 8: 1569-1581

$15 \mathrm{Ni}$ X, Li Y, Zhang Y et al. Enteral nutrition fecal microbiota transplantation via percutaneous endoscopic cecostomy in treatment of recurrent ulcerative colitis. Parenter Enteral Nutr 2015; 22: 29- 38 (In Chinese) 